\title{
Estimation of the amplitude of dynamic stresses on the contour of the well in the mountain range by the parameters of bench tests of hydraulic oscillators
}

\author{
Oleh Usov, ${ }^{1, *}$ \\ ${ }^{1}$ Institute of Geotechnical Mechanics named by N. Poljakov of National Academy of Sciences of \\ Ukraine, 49005, Dnipro, Simferopolska Str., 2a, Ukraine
}

\begin{abstract}
Presented a method for calculating the dynamic tangential stresses around the wellbore, at the time of its treatment by the hydraulic pulse generator, by the values of its characteristics, obtained at the bench test, and the frequency coefficients of influence. Criteria are formulated for selecting the natural frequencies of the measuring sensors that ensure the coincidence of voltages in the array and on the measuring bench.
\end{abstract}

\section{Introduction}

For the intensification of technological processes (oil and gas recovery, discharge of coal beds from excessive stresses, etc.), well treatment technologies are used with various generators of hydraulic oscillations or pulses. It is almost impossible to measure the dynamic stresses arising in the rock mass near the borehole contour when the hydraulic oscillator is operating. They are estimated by calculation methods.

Reliable calculated stress assessment in case of forced oscillations of a complex "generator - liquid in a well - rock mass" system is possible only if the oscillation parameters - the frequency and amplitude of movement of the generator - are known. These parameters can be accurately determined only for generators of the mechanical or electromechanical type [1].

And the exact parameters of the generators based on hydrodynamic, electro-hydraulic, cavitation or shock effects are not known in advance [2]. They are determined by the calculated processing of the results of bench test measurements in the well simulator. In this case, the calculation of the characteristics of the generator and the calculation of the dynamic stresses in the rock mass from these characteristics is a very difficult and timeconsuming task.

Therefore, the search for criteria for selecting the parameters of measuring sensors for a reliable assessment of dynamic stresses in a rock mass according to the results of bench tests of hydraulic oscillations (pulse) generators without complex calculations is an important task.

The idea of work is to consider a deformation sensor and a borehole drilled in a rock

*Corresponding author: igtm@ukr.net 
mass as single-type oscillatory systems having different natural frequencies.

Formulation of the problem. Using reliable methods of the theory of oscillations, to estimate the ratio of the amplitudes of forced oscillations of two systems of the same type with different natural frequencies.

\section{Methods}

In all classical courses in the theory of oscillations, the problem of forced oscillations of the simplest deformation sensor in the form of a spring-loaded mass on a weightless spring under the action of the driving force $F(t)$, represented as a decomposition into a complex Fourier series, is considered:

$$
F(t)=\sum_{j} F_{j} \cdot e^{i \cdot j \cdot \Theta \cdot t}
$$

where: $F_{j}$ - decomposition factors of the driving force $F(t) ; i$ - imaginary unit; $j$ summation index; $\Theta$ is the base frequency of decomposition, inversely proportional to the period of complex oscillations of the external force; $t$ - time.

The solution to this problem is (formula (2.57) from [3])

$$
W(t)=\sum_{j} \frac{F_{j}}{m} \cdot \beta_{j} \cdot e^{i \cdot j \cdot \Theta \cdot t}
$$

where: $W(t)$ is the displacement of the sensitive element of the sensor (spring); $m$ sensor mass; $\beta_{j}$ - the harmonic coefficient of influence of the frequency, depending on the natural frequency of the sensor $\omega_{S}$, the attenuation coefficient equals zero, and the frequency of forced oscillations.

$$
\beta_{j}=\frac{1}{\omega_{S}{ }^{2}-j^{2} \Theta^{2}}
$$

For other types of sensors - rod, membrane, ring - the expressions for $\beta_{j}$ will be different, but they will still be determined by the parameters $\omega_{S}, \Theta$. This circumstance is important for further discussion.

Formula (2) allows to solve not only the direct, but also the inverse problem - the components of the decomposition of the acting force $F_{j}$ can be determined from the decomposition components of the displacement of the sensitive element of the sensor (spring) $F_{j}$ :

$$
\sum_{j} W_{j} \cdot e^{i \cdot j \cdot \Theta \cdot t}=\sum_{j} \frac{F_{j}}{m} \cdot \beta_{j} \cdot e^{i \cdot j \cdot \Theta \cdot t}
$$

Comparing the members with the same indexes $j$ in the left and right parts (3), we obtain:

$$
W_{j}=\frac{F_{j}}{m} \beta_{j}
$$

or

$$
F_{j}=\frac{W_{j}}{\beta_{j}} m
$$


Multiplying both sides of (5) by $e^{i \cdot j \cdot \Theta \cdot t}$, performing summation and replacing the sum on the left side with the symbol $F(t)$ by the formula (1), we obtain

$$
F(t)=\sum_{j} W_{j} \frac{m}{\beta_{j}} e^{i \cdot j \cdot \Theta \cdot t}
$$

The right-hand side of the last expression includes the mass $\mathrm{m}$, which is related to the natural frequency of the sensor $\omega_{S}$ and the stiffness of its spring $k$ by the well-known expression

$$
m=\frac{k}{\omega_{S}^{2}}
$$

and substituting $\beta_{j}$ from (3) into it, we obtain

$$
F(t)=\sum_{j} W_{j} \cdot k \cdot\left(1-j^{2} \frac{\Theta^{2}}{\omega_{S}{ }^{2}}\right) \cdot e^{i \cdot j \cdot \Theta \cdot t}
$$

The product of the first two factors on the right side of formula (8) is the amplitude of the forces in the spring - sensitive element of the sensor $F_{S j}$ corresponding to the $j$ component of the series decomposition. This directly follows from the proportionality of the displacements to the inner forces in the solid body. Moreover, the formula

$$
W_{j} \cdot k=F_{S j}
$$

valid not only for the spring, but also for any other deformation sensor.

Substituting (9) into (8) and entering the value

$$
\beta_{S j}^{*}=1-\frac{j^{2} \Theta^{2}}{\omega_{S}{ }^{2}}
$$

we obtain

$$
F(t)=\sum_{j} F_{S j} \cdot \beta_{S j}^{*} \cdot e^{i \cdot j \cdot \Theta \cdot t}
$$

Formula (11) makes it possible to determine the unknown components of the external force $F(t)$ from the results of bench test measurements of the components of the forces arising in the sensitive element of the deformation sensor $F_{S j}$.

Moreover, formula (11) makes it possible to determine by calculation the components of the internal forces arising under the action of the same external force $F(t)$ of any sensor with a different natural frequency, including the rock mass itself. To do this, it suffices to write (11) for a rock mass with the same forced oscillation frequency $j \Theta$, natural frequency $\omega_{R}$ and unknown components of its forces $F_{R j}$

$$
F(t)=\sum_{j} F_{R j} \cdot \beta_{R j}^{*} \cdot e^{i \cdot j \cdot \Theta \cdot t}
$$

and equate the right sides (12) and (11). Will get

$$
\sum_{j} F_{R j} \cdot \beta_{R j}{ }^{*} \cdot e^{i \cdot j \cdot \Theta \cdot t}=\sum_{j} F_{S j} \cdot \beta_{S j}{ }^{*} \cdot e^{i \cdot j \cdot \Theta \cdot t}
$$

Comparing each member of the expansion in the left and right parts, we obtain 


$$
F_{R j}=F_{s j} \frac{\beta_{S j}{ }^{*}}{\beta_{R j}{ }^{*}}
$$

The components of the internal forces of the deformation sensor $F_{S j}$ are determined from the results of static calibration using formula (9). Writing it down for moving $W_{T A R}$, under the action of a constant calibrating force $F_{T A R}$

$$
W_{T A R} \cdot k=F_{T A R}
$$

and dividing (9) by (15), we obtain

$$
F_{S j}=F_{T A R} \frac{W_{j}}{W_{T A R}}
$$

The accuracy of determining $F_{S j}$ is very high, since it is determined by the accuracy of measuring the static calibration load $F_{T A R}$, the corresponding static displacement $W_{T A R}$ and the current dynamic displacement $W_{j}$.

Substituting $F_{S j}$ from (16) into (14) and summing up the components $F_{R j}$, we obtain an expression for the characteristic internal forces in the rock mass $F_{R}(t)$

$$
F_{R}(t)=\frac{F_{T A R}}{W_{T A R}} \sum_{j} W_{j} \frac{\beta_{S j}{ }^{*}}{\beta_{R j}{ }^{*}} e^{i \cdot j \cdot \Theta \cdot t}
$$

where: $F_{T A R}$ - the value of the static calibration load of the sensor; $W_{T A R}$ - displacement of the sensitive element of the sensor regestered by itself under the static calibration load; $\beta_{S j}{ }^{*}, \beta_{R j}{ }^{*}$ - frequency coefficients of influence for the sensor and rock mass, determined for the spring-loaded mass without taking the attenuation into account by formula (10); $i$ imaginary unit; $j$ - the summation index; $\Theta$ - basic decomposition frequency.

The resulting calculation formula has a universal character. Since our task is to study the stresses in the rock mass resulting from hydraulic impulses, the symbol $F_{T A R}$ should be understood as the value of the static calibration pressure, and the symbol $F_{R}(t)$ means the stresses in the rock mass. At the same time, the circumferential (tangential) stresses arising on the well contour should be chosen as the characteristic stresses of the rock mass. By their nature, they coincide with the tangential stresses of the pipe - simulator of a well, pasted over with strain gauge sensors, in a direction perpendicular to its axis.

\section{Results and discussion}

The most important thing is to correctly determine the size of the well simulator. To do this, we analyze the expression in the brackets of the right-hand side of (17), which determines the stress difference in the rock mass and the one from the bench test. For the spring sensor without oscillation attenuation, it is determined by the formula (10)

$$
\frac{\beta_{S j}{ }^{*}}{\beta_{R j}{ }^{*}}=\frac{1-j^{2} \frac{\Theta^{2}}{\omega_{S}{ }^{2}}}{1-j^{2} \frac{\Theta^{2}}{\omega_{R}{ }^{2}}}
$$


Analyzing (18) it is easy to notice that if the highest significant frequency $j \Theta$ of the source of oscillations is much less than the natural frequencies of both the sensor $\omega_{S}$ and the mass $\omega_{R}$, then the ratio $\beta_{S j}{ }^{*} / \beta_{R j}{ }^{*} \rightarrow 1$. Which means that the stresses in the rock mass will almost coincide with the stresses in the walls of the pipe - simulator.

This circumstance allows us to identify the stresses in the rock mass with the stresses in the sensitive element of the sensor in the range of significant frequencies of decomposition $\sim 100-200 \mathrm{~Hz}$. The stresses in the rock mass are determined by the formula (17), in which $\beta_{S j}{ }^{*} / \beta_{R j}{ }^{*}=1$ :

$$
F_{R}(t)=\frac{F_{T A R}}{W_{T A R}} \sum_{j} W_{j} \cdot e^{i \cdot j \cdot \cdot \cdot t}
$$

For higher frequencies this option is not applicable. With comparable frequencies of the oscillation source $j \Theta$ and the rock mass $\omega_{R}$, the calculation of the relationship $\beta_{S j}{ }^{*} / \beta_{R j}{ }^{*}$ will be very laborious, since for the pipe and well these coefficients are not expressed through elementary functions. In addition, it is not possible to adequately take into account the attenuation coefficient.

A partial way out of this situation can be the composition of a correlation dependence of $\beta_{S j}{ }^{*}$ on the natural frequencies of the same-type measuring sensors (at least three of them are needed). However, such an approach does not eliminate the error associated, firstly with the extrapolation accuracy of the correlation curve to the oscillation frequency of the rock mass, and secondly, with the possible presence of extreme points not considered in its composition.

Another case of coincidence of stresses in the rock mass with the stresses in the sensitive element of the sensor takes place at the same frequencies of their own oscillations. When $\omega_{S}=\omega_{R}$, the ratio $\beta_{S j}{ }^{*} / \beta_{R j}{ }^{*}$ is also $\rightarrow 1$. And the attenuation coefficient without large error can be taken to be the same in the expressions for $\beta_{S j}{ }^{*}$ and $\beta_{R j}{ }^{*}$.

The condition of coincidence of the frequencies of the natural oscillations of the sensor and the rock mass is provided as follows. Geophysical methods of well investigation, or other methods, determine the intrinsic frequencies of the rock. According to the values of the natural frequencies of the array $\omega_{R}$, the sensor is selected. The natural frequency of the sensor should correspond to the natural frequency of the rock mass $\omega_{S} \approx \omega_{R}$. With a small difference between the frequencies $\omega_{S}$ and $\omega_{R}$, the stresses in the rock mass $F_{R}(t)$ are quite accurately determined by formula (17).

If it was not possible to pick up the sensor with the necessary natural frequency, then it makes sense to use the pipe itself, which simulates a well during bench tests instead of the ready sensor.

To do this, it is necessary to register the displacements of the external wall of the pipe inside which the generator is placed with the help of strain gauge transducers. The required frequency of natural oscillations of the pipe is provided by the selection of its diameter, wall thickness and material of which it is made. The stresses in the rock mass $F_{R}(t)$ are determined by formula (19) according to the readings of the strain gauge sensors.

And finally, if the lowest significant frequency $j \Theta$ of the oscillation source is much more than the natural frequencies of both the sensor $\omega_{S}$ and the rock mass $\omega_{R}$, then formula (18) takes the form

$$
\frac{\beta_{S j}{ }^{*}}{\beta_{R j}{ }^{*}}=\frac{\omega_{R}{ }^{2}}{\omega_{S}{ }^{2}}
$$

And the stresses in the array $F_{R}(t)$ are determined by the formula (17) taking into account (20) 


$$
F_{R}(t)=\frac{\omega_{R}{ }^{2}}{\omega_{S}{ }^{2}} \frac{F_{T A R}}{W_{T A R}} \sum_{j} W_{j} \cdot e^{i \cdot j \cdot \Theta \cdot t}
$$

The latter formula is relevant for ultrasonic generators in which the lowest significant frequency $\sim 20000 \mathrm{~Hz}$ is much higher than the frequencies of the natural oscillations of the rock mass.

\section{Conclusions}

Reliable assessment of dynamic stresses in the rock mass, when the well is treated by a hydraulic oscillation (pulse) generator according to the parameters of its bench tests, is achieved when one of the three conditions is fulfilled:

1) If the highest significant frequency $j \Theta$ of the source of oscillations is much less than the natural frequencies of both the sensor $\omega_{S}$ and the rock mass $\omega_{R}$. Stresses are determined by formula (19).

2) If the natural frequency of the measuring sensor corresponds to the natural frequency of the rock mass $\omega_{S} \approx \omega_{R}$. The stresses are also determined by formula (19).

3 ) If the lowest significant frequency $j \Theta$ of the source of oscillations is much more than the natural frequencies of both the sensor $\omega_{S}$ and the rock mass $\omega_{R}$. Stresses are determined by formula (21).

\section{References}

1. Poturaiev V.N., Minieiev S.P. (1993). Pulsatsionnye $i$ volnovye effekty $v$ hornom massive. Kyiv: Naukova dumka

2. Usov, O.A, Potapenko, A.A. (2013). Sravnenie energii vozdeystviya kavitatsionnyh kolebaniy vody na stenki skvazhiny s energiey udara otboynogo molotka po massivu gornyh porod. Geotechnicheskkaya mechanika, (108), 223-229

3. Babakov M.I. (1968). Soprotivlenie materialov. Moskva: Nauka 